\title{
Efficient Oligo nucleotide mediated CRISPR-Cas9 Gene Editing in Aspergilli
}

Nødvig, Christina Spuur; Hoof, Jakob Blæsbjerg; Kogle, Martin Engelhard; Jarczynska, Zofia Dorota; Lehmbeck, Jan; Klitgaard, Dorte K; Mortensen, Uffe Hasbro

\section{Published in:}

Fungal Genetics and Biology

Link to article, DOI:

10.1016/j.fgb.2018.01.004

Publication date:

2018

Document Version

Peer reviewed version

Link back to DTU Orbit

Citation (APA):

Nødvig, C. S., Hoof, J. B., Kogle, M. E., Jarczynska, Z. D., Lehmbeck, J., Klitgaard, D. K., \& Mortensen, U. H. (2018). Efficient Oligo nucleotide mediated CRISPR-Cas9 Gene Editing in Aspergilli. Fungal Genetics and Biology, 115, 78-89. https://doi.org/10.1016/j.fgb.2018.01.004

\section{General rights}

Copyright and moral rights for the publications made accessible in the public portal are retained by the authors and/or other copyright owners and it is a condition of accessing publications that users recognise and abide by the legal requirements associated with these rights.

- Users may download and print one copy of any publication from the public portal for the purpose of private study or research.

- You may not further distribute the material or use it for any profit-making activity or commercial gain

- You may freely distribute the URL identifying the publication in the public portal 


\section{Accepted Manuscript}

Tools and techniques

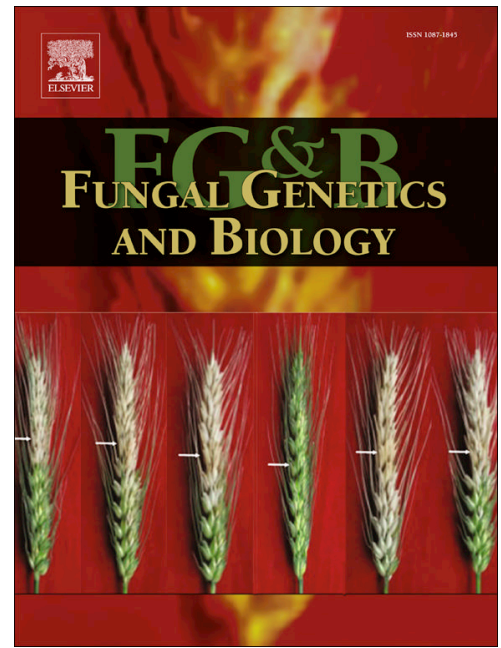

Efficient Oligo nucleotide mediated CRISPR-Cas9 Gene Editing in Aspergilli

Christina S. Nødvig, Jakob B. Hoof, Martin E. Kogle, Zofia D. Jarczynska, Jan

Lehmbeck, Dorte K. Klitgaard, Uffe H. Mortensen

PII:

S1087-1845(18)30004-5

DOI: https://doi.org/10.1016/j.fgb.2018.01.004

Reference: YFGBI 3107

To appear in: $\quad$ Fungal Genetics and Biology

Received Date: $\quad 26$ September 2017

Revised Date: $\quad 5$ January 2018

Accepted Date: 6 January 2018

Please cite this article as: Nødvig, C.S., Hoof, J.B., Kogle, M.E., Jarczynska, Z.D., Lehmbeck, J., Klitgaard, D.K., Mortensen, U.H., Efficient Oligo nucleotide mediated CRISPR-Cas9 Gene Editing in Aspergilli, Fungal Genetics and Biology (2018), doi: https://doi.org/10.1016/j.fgb.2018.01.004

This is a PDF file of an unedited manuscript that has been accepted for publication. As a service to our customers we are providing this early version of the manuscript. The manuscript will undergo copyediting, typesetting, and review of the resulting proof before it is published in its final form. Please note that during the production process errors may be discovered which could affect the content, and all legal disclaimers that apply to the journal pertain. 


\section{Efficient Oligo nucleotide mediated CRISPR-Cas9 Gene Editing in Aspergilli}

Christina S. Nødvig ${ }^{a}$, Jakob B. Hoof ${ }^{a}$, Martin E. Kogle ${ }^{a}$, Zofia D. Jarczynska ${ }^{a}$, Jan Lehmbeck ${ }^{b}$,

Dorte K. Klitgaard ${ }^{c}$, \& Uffe H. Mortensen ${ }^{a^{*}}$

a Department of Biotechnology and Biomedicine, Technical University of Denmark, Søltofts Plads, Building 223, Kongens Lyngby, 2800, Denmark.

${ }^{\mathrm{b}}$ Department of Fungal Strain Technology, Novozymes A/S, Bagsværd, 2880, Denmark.

${ }^{\mathrm{C}}$ Department of Protein Diversity, Novozymes A/S, Bagsværd, 2880, Denmark.

* To whom correspondence should be addressed. Tel: +45 4525 2701; Fax: +45 4588 4148; Email: um@bio.dtu.dk.

\section{ABSTRACT}

CRISPR-Cas9 technologies are revolutionizing fungal gene editing. Here we show that survival of specific Cas9/sgRNA mediated DNA double strand breaks (DSBs) depends on the non-homologous end-joining, NHEJ, DNA repair pathway and we use this observation to develop a tool to assess protospacer efficiency in Aspergillus nidulans. Moreover, we show that in NHEJ deficient strains, highly efficient marker-free gene targeting can be performed. Indeed, we show that even single-stranded oligo nucleotides efficiently works as repair templates of specific Cas9/sgRNA induced DNA DSBs in A. nidulans, A. niger, and in A. oryzae indicating that this type of repair may be wide spread in filamentous fungi. Importantly, we demonstrate that by using single-stranded oligo nucleotides for CRISPR-Cas9 mediated gene editing it is possible to introduce specific point mutations as well gene deletions at efficiencies approaching $100 \%$. The efficiency of the system invites for multiplexing and we have designed a vector system with the capacity of delivering Cas9 and multiple sgRNAs based on polymerase III promoters and tRNA spacers. We show that it is possible to introduce two point mutations and one gene insertion in one transformation experiment with a very high efficiency. Our system is compatible with future high-throughput gene-editing experiments. 


\section{Introduction}

Filamentous fungi constitute a rich source of enzymes and bioactive metabolites for the biotech-, food- and pharma industries and only a tiny fraction of these activities have been identified and characterized (1-4). At the same time fungi may also produce harmful mycotoxins and act as undesirable, and even deadly, plant-, animal- and, human pathogens $(2,5)$. Towards a more efficient exploitation of the fungal product potential as well as to better understand fungal biology to combat disease, genomes of numerous species have been fully sequenced; and this number is rapidly increasing $(6,7)$. Hence, the number of known genes that putatively encode proteins with potentially interesting enzymatic properties, genes that putatively are involved in production of secondary metabolites, and genes that putatively may be involved in pathogenicity is enormous. However, the route from bioinformatic gene predictions towards understanding true functions of gene products has been hampered by lack of tools to perform rapid reverse genetics in filamentous fungi (8). With the implementation of CRISPR-Cas9 genome editing technologies in fungi this is quickly changing (9-14). RNAguided Cas9 endonuclease can easily be programmed to cleave virtually any DNA sequence with a very high degree of specificity; and subsequent repair of the resulting DNA double-strand breaks (DSBs) can be employed as a basis for site specific mutagenesis mediated by non-homologous end-joining, or, for the introduction of specific DNA changes via HR mediated gene targeting $(9,15-19)$.

We have previously presented a system that allows for efficient fungal CRISPR mediated gene editing (9). This system is based on Streptococcus pyogenes Cas9, which have been adapted to fungal use by employing a copy of cas9, which is codon optimized for production in Aspergillus niger and extended by a sequence that adds an SV40-NLS to the C-terminus of the resulting enzyme. Production of the sgRNA is achieved by expressing a gene that is controlled by an RNA polymerase II promoter. For that reason, the sgRNA is embedded in a splicing cassette as it is flanked by the hammerhead $(\mathrm{HH})$ and hepatitis delta virus (HDV) ribozymes. Hence, the mature sgRNA is released from the transcript by the action of these to ribozymes. To facilitate fungal CRISPR technology, we made an AMA1 based vector set where the individual vectors contain selectable markers and where the cas9 and sgRNA genes can be easily inserted by seamless cloning strategies. Using this system we have performed CRISPR mediated gene editing in seven different fungal species $(9,20)$.

Our current system is designed for introducing individual gene alterations in fungi and is not well suited for multiplexing and automated genetic engineering. The main limitations are evaluation of sgRNA efficiency, labor intense work to generate gene-targeting substrates (GTSs), and production of multiple different sgRNA species in a single experiment. In the present study, we investigate the role of non-homologous end-joining in sealing Cas9 mediated DNA DSBs, and we use the insights to establish a tool to assess protospacer efficiency, TAPE, in filamentous fungi. Moreover, we demonstrate that single-stranded oligonucleotides can be efficiently used as GTSs to introduce specific deletions and point mutations via repair of specific Cas9 induced DNA DSBs; hence, eliminating the need for construction of complex GTSs; a finding that sets the stage for simple high- 
throughput genetic engineering in filamentous fungi. Lastly, by exploiting a polymerase III promoter and tRNA sequences as spacers for the release of multiple different sgRNAs, we demonstrate that our new tools can be used for efficient fungal marker-free multiplexing gene-editing experiments.

\section{Materials and methods}

\subsection{Strains and media}

The Aspergillus strains used for CRISPR-Cas9 gene editing experiments are listed in Table 1. Escherichia coli DH5 $\alpha$ was used as host for propagating all plasmids using solid, 2\% agar, or liquid LB medium containing 100 $\mu \mathrm{g} / \mathrm{ml}$ ampicillin as growth medium. All fungal strains were cultivated on solid minimal media (MM): 1\% glucose, $1 x$ nitrate salt solution (21), $0.001 \%$ Thiamine, $1 x$ trace metal solution (22), $2 \%$ agar. When required MM was supplemented with $10 \mathrm{mM}$ uridine (Uri), $10 \mathrm{mM}$ uracil (Ura), and/or $4 \mathrm{mML}$-arginine (Arg). For transformation, transformation medium (TM) was used. TM is MM where glucose is replaced with $1 \mathrm{M}$ sucrose.

\subsection{Genomic DNA, PCR and vector construction}

Primer design was based on the $A$. nidulans and $A$. niger genome sequences, which are publicly available through AspGD/FungiDB (version 5) and JGI (version 4.0), respectively $(23,24)$. Fungal genomic DNA (gDNA) for PCR reactions was isolated via FastDNA SPIN Kit for Soil DNA extraction kit (MP Biomedicals, USA) using the instructions of the manufacturer. All vectors were constructed by USER-cloning and -fusion (25, 26); and PfuX7 (27) was used as polymerase to generate all PCR fragments for vector construction. A list of all primers (Integrated DNA Technologies (IDT), US) used in this study is provided in Table S1. Detailed information concerning how to construct CRISPR vectors for ribozyme based in vivo sgRNA release is described in Nødvig et al (9). Construction of CRISPR-tRNA vectors designed for RNase $P$ and RNase $Z$ in vivo catalyzed sgRNA release is explained in section 3.5. For primer design for construction of primers for CRISPR-tRNA vectors, see Fig. S1. Here we explain how protospacers, through primer tails, can be inserted between promoter and sgRNAterminator fragments. For construction of CRISPR vectors containing $A f_{-} U 6-1 p$ and $A f_{-} U 3 p$ promoters, we obtained fragments by PCR using gDNA from Aspergillus fumigatus Af293 as template using primer pairs CSN440+CSN441 and CSN438+CSN439, respectively The tRNA spacer-based sgRNA expression vector (pFC902) was constructed by fusing two DNA fragments (gBlocks, IDT) by USER fusion using pFC330 as vector fragment, see Fig. S2 for details. pFC902 has been deposited in the Addgene collection. The resulting plasmid pFC902 was used as PCR template for all subsequent CRISPR-tRNA vectors. Details concerning all CRISPR vectors, including the mechanism of sgRNA release, is presented in Table S2. gDNA extracted from NID5 was used as template for amplifying the putative $A$. nidulans U6 promoter and targeting fragments for construction of GTS vectors for $A$. nidulans (Table S3), while the sgRNA backbone, the HDV ribozyme and the trpC terminator were amplified from pFC334. pU0002 (26) was used as vector backbone for construction of all marker-free GTS vectors. GTS-kusA-2000, which contains AfpyrG flanked by direct repeats, was constructed by using pU2002A (24) as vector backbone and PCR derived targeting sequences amplified from NIG1 gDNA for kusA deletion in $A$. 
niger (Fig. S3, Table S1). Finally, construction details of the AMA1 vectors for analysis of promoter strength are specified in Table S4.

\subsection{Fungal transformation, protospacer, and strain validation}

Preparation of fungal protoplasts and transformation were performed as previously described (28). Transformants were obtained on solid TM medium with or without supplements. In gene-targeting experiments, $1 \mu \mathrm{g}$ of CRISPR vector was used in combination with either $1 \mu \mathrm{g}$ of a linear or circular GTS when the GTS was selected via a marker gene, or $2 \mu \mathrm{g}$ if there was no selection for the GTS, in a total volume of 150 $\mu \mathrm{l}$ of PCT buffer (9). In gene-editing experiments, $10 \mu \mathrm{L}$ of $100 \mu \mathrm{M}$ stock solutions of oligo nucleotides (GEOligos, see Table S5) were added instead of a GTS serving as repair templates. Strain validation was performed by diagnostic PCR reactions using the strategy outlined in in Nødvig et al (9). We used a t-test $(P<0.05$; twotailed; paired) in Excel (Microsoft) for evaluating protospacer efficiency.

\subsection{Fluorescence microscopy}

Microscopy was essentially performed as previously described (29). Images were captured with a cooled Evolution QEi monochrome digital camera (Media Cybernetics Inc.) mounted on a Nikon Eclipse E1000 microscope using a 10X ocular. Images of spores were acquired using 100X or 20X objectives.

\section{Results}

3.1. A technique to assess protospacer efficiency, TAPE, identifies sgRNAs for efficient marker-free genedeletions

We have previously demonstrated that Cas9/sgRNA nucleases are able to generate mutations in filamentous fungi at specific chromosomal sites that are complementary to the protospacer of the sgRNA (9); and we speculated that these mutations were the result of error-prone repair of Cas 9 induced DNA DSBs by the NHEJ pathway. To test this hypothesis we individually transformed four different $p y r G$-based CRISPR plasmids (pFC897, pFC934, pFC937, and pFC920) each containing AMA1 and cas9 as well as one of four sgRNA genes, yA-sgRNA1-4, into NHEJ deficient (NID1) and proficient (NID5) A. nidulans strains in three independent trials. Each of these protospacers target Cas9 to different positions within yA (see Fig. 1A), which encodes a laccase converting the yellow spore compound YWA1 into green pigment (30). Successful mutagenesis of yA by Cas9 can easily be scored as colonies displaying yellow conidia versus wild-type green conidia, as we demonstrated previously (10). The sgRNA genes on the CRISPR vectors are transcribed by Polymerase II and mature sgRNAs are released by ribozymes contained in the full-length transcripts $(9,31)$. As a reference experiment, NID1 and NID5 were also transformed in parallel with an empty pyrG-based CRISPR plasmid using the same batch of protoplasts. 
When pyrG-based CRISPR plasmids expressing yA-sgRNA2 or yA-sgRNA3 were transformed into NHEJ proficient NID5 strains; all resulting transformants displayed a healthy morphology including the ability to form conidia (Fig. 1B). Moreover, the numbers of transformants obtained with these vectors were similar to the numbers of transformants obtained with the corresponding empty CRISPR vector (Fig. 1C). In contrast, with the pyrG-based CRISPR plasmids expressing $y A$-sgRNA1 and $y A-s g R N A 4$, the majority of the transformants with NID5 strains appeared crippled showing no, or severely impaired, conidiation. Moreover, the numbers of transformants with a healthy morphology were significantly decreased, 7- and 4- fold, respectively, relative to the number obtained with the empty CRISPR vector. These effects were even more pronounced with NHEJ deficient NID1 strains where transformations with CRISPR plasmids expressing yA-sgRNA1 and yA-sgRNA4 generated almost no healthy transformants. As a result, the numbers of transformants obtained with these plasmids were further reduced, around 5- and 8-fold respectively, relative to the corresponding numbers obtained with the empty vector, and these reductions were also significant (Fig. 1C). As a result the total decrease in the numbers of transformants obtained with pyrG-based CRISPR plasmids expressing yA-sgRNA1 and $y A$-sgRNA4 with NID5 strains were reduced more than 30 -fold compared to the numbers obtained with the empty CRISPR vector. These results strongly indicate that with $y A-s g R N A 1$ and $y A-s g R N A 4$ potentially lethal Cas9 DNA DSBs are induced at sufficiently efficient rates to reduce survival of transformants significantly; and that functional NHEJ is important for repairing these breaks to increase the chance of survival. In further support of NHEJ repair playing an important role in the repair of Cas9/sgRNA induced DNA DSBs, we find that with NID5, but not with NID1, conidiating transformants obtained with CRISPR plasmids expressing yA-sgRNA1 and $y A-s g R N A 4$ readily developed healthy yellow colonies when spores from these transformants were restreaked on solid medium selecting for the pyrG-based CRISPR plasmid (Fig. S4A-C). These results indicate that DSBs in $y A$ were joined by error prone NHEJ repair to create mutations (9).

In similar experiments with the yeast Saccharomyces cerevisiae, we also found that reduced numbers of transformants are a consequence of lethal unrepaired Cas9 induced DNA double strand breaks (32). Moreover, we used this observation to develop a technique to assess protospacer efficiency (TAPE) exploiting that sgRNAs that efficiently induce cell death in the absence of a repair template also stimulate efficient gene targeting. If, the TAPE concept is also valid for A. nidulans, TAPE would predict that $y A-s g R N A 1$ and $y A-s g R N A 4$ stimulate gene targeting into the $y A$ locus more efficiently than $y A-s g R N A 2$ and $y A-s g R N A 3$. To address this possibility, we individually co-transformed NHEJ deficient NID1 strains with the four pyrG-based CRISPR plasmids expressing $y A-s g R N A 1-4$ along with a circular marker-free $y A$ GTS (cGTS-yA-2000) designed for deleting $y A$ by marker-free gene-targeting as it contains a $4 \mathrm{~kb}$ insert composed by a fusion of $2 \mathrm{~kb}$ of up-and 2 $\mathrm{kb}$ of down-stream $y A$ sequences. In agreement with the TAPE prediction, the vast majority of the transformants obtained with the two pyrG-based CRISPR plasmids expressing $y A-s g R N A 1$ and $y A-s g R N A 4$ formed yellow conidia when the cGTS-yA-2000 was included in the transformation experiment (Fig. 1B). Moreover, the numbers of transformants obtained with CRISPR vectors expressing yA-sgRNA1 and yA-sgRNA4 were significantly increased by around 20- and 16-fold, respectively, as compared to the corresponding numbers obtained in the absence of this substrate (Fig. 1C). Moreover, diagnostic PCR analyses of 12 yellow 
colonies obtained from these experiments demonstrated that they all contained a deletion of $y A$ (Fig. S5A and S5B). In contrast, formation of yellow transformants obtained with pyrG-based CRISPR plasmids expressing $y A$ sgRNA2 and $y A-s g R N A 3$ were rare, Fig. 1B. Together these results strongly suggest that TAPE can be used to predict sgRNA efficiency, and that sgRNAs selected by TAPE can be used to perform efficient marker-free genedeletions in NHEJ deficient strains.

3.2. CRISPR plasmid stability and promoter strength of cas9 and sgRNA influence CRISPR mediated genetargeting efficiency

We next compared the targeting efficiency of linear and circular GTSs for marker-free deletion of $y A$ (Table 2). Specifically, GTSs containing different lengths of targeting sequences were co-transformed into the NHEJ deficient strain NID1 with $y A$-sgRNA1 encoding CRISPR vectors based on either pyrG (pFC897) or $\arg B(\mathrm{pFC} 334)$ markers, and plated on solid medium selecting for either pFC897 or pFC334. With all, GTSs, homogenously yellow transformants were frequently formed, Table 2 . This phenotype is due the fact that all conidia were yellow, and such transformants most likely result from events where the $y A$ disruption happened early after transformation. Based on the fact that NID1 transformed with pFC334 (Fig. 1B) did not produce healthy homogenously yellow transformants in the absence of a GTS, we envisioned that the homogenously yellow colonies formed in the presence of GTSs are predominantly formed as the result of successful deletion of the yA gene. We substantiated this conclusion by demonstrating that five randomly selected yellow colonies generated by the GT substrates with the shortest targeting sequences, $200 \mathrm{bps}$, contained the expected yA deletion as judged by diagnostic PCR analyses (Fig. S5C).

With the $\arg B$-based $y A$-sgRNA1 encoding CRISPR vector ( $\mathrm{pFC} 334$ ) the percentage of yellow transformants within the total population of transformants were always higher with circular GTSs as compared to the corresponding linear GTSs. The highest efficiencies, > 90\%, were obtained with circular GTSs containing 1000 and 2000 bps yA targeting sequences. With both circular- and linear GTSs, yellow colonies were obtained with low efficiencies, $<15 \%$, when the targeting sequences were reduced to $200 \mathrm{bps}$. In contrast, gene targeting induced by expressing yA-sgRNA1 from a pyrG-based CRISPR plasmid (pFC897) appeared less sensitive to reductions in the length of $y A$ targeting sequences and to the design of the GTS. Hence, all circular and linear substrates containing $y A$ targeting sequences $\geq 500$ bps produced yellow colonies with high efficiencies as > $90 \%$ of the colonies in the total population of transformants were yellow. The design of the GTS influenced the results only with short 200 bps $y A$ targeting sequences as the gene-targeting efficiencies dropped to around $75 \%$ and $17 \%$ in the case of the circular and linear GTSs, respectively. The higher efficiencies obtained with pyrG based CRISPR plasmids could be due to a higher plasmid copy number and/or plasmid stability, and there 
by higher and more uniform expression levels of the cas 9 and sgRNA genes when pyrG, rather than $\arg B$, is used as selection marker.

To investigate whether expression levels vary between AMA1 based plasmids selected by either $p y r G$ or $\arg B$ we constructed a set of MRFP expression plasmids, see Table S4, and transformed them individually into NID1. Specifically, we tested expression of an mRFP gene under the control of either the tef1- or the gpdA promoter, utilized for cas9 and sgRNA expression, respectively, in our CRISPR vectors, by visualizing the mRFP signal in conidiospores by fluorescence microscopy, Fig. 2 and Fig. S6A - S6C. With conidia obtained from a NID1 strain transformed with pyrG based vectors we observed little variation in the RFP signal between individual spores. In contrast, the mRFP signal varied considerably between individual spores obtained from strains containing $\arg B$ selected plasmids. Moreover, relative to the mRFP signal levels in conidia obtained from strains containing pyrG based vectors, the mRFP signal levels were generally much lower in conidia obtained from strains containing the corresponding $\arg B$ vectors. However, a few of these spores contained similar or even higher mRFP signals as compared to the signals obtained with spores from strains containing pyrG vectors. Hence, the higher gene-targeting efficiency observed with NID1 strains transformed with pyrG-based CRISPR plasmids may be due to a higher number of nuclei that contain sufficient Cas 9 and sgRNA levels to support efficient DNA DSB formation as compared to strains transformed with $\arg B$-based CRISPR plasmids. Interestingly, we also note that the tef1 promoter is considerably stronger than the gpdA promoter as 14 times longer exposure times of spores containing plasmids expressing $m R F P$ under the control of the gpdA promoter were required to achieve signal strengths comparable to that observed with spores expressing RFP under the control of the TEF1 promoter, Fig. S6A - S6B.

\subsection{Efficient single-stranded oligonucleotide mediated CRISPR gene-editing in A. nidulans}

The fact that significant Cas9 induced gene targeting can be achieved with GTSs containing short targeting sequences inspired us to examine whether short single-stranded oligonucleotides can be used as a repair template for accurate Cas9 induced gene editing in A. nidulans. Moreover, since single-stranded gene-editing oligonucleotides (GE-oligonucleotides) can be designed to interact with the sense or the antisense strand of transcribed loci, we tested whether GE-oligonucleotides exhibit strand preference for repair. Accordingly, a pyrG-based CRISPR plasmid expressing yA-sgRNA1 (pFC897) was co-transformed with two different 90-mer gene-editing oligonucleotides, GE-Oligo1 and GE-Oligo2, which both were designed to introduce a specific AAC->TAA non-sense mutation in $y A$ next to the Cas9/yA-sgRNA1 cleavage site in $y A$. In both cases, the mutagenic codon is located in the center of the gene-editing oligonucleotide, but GE-Oligo1 is complementary to the anti-sense strand and GE-Oligo2 to the sense strand of $y A$ (Fig. S7A).

With GE-Oligo1 and GE-Oligo2 we obtained 10 and 20 transformants and 11 and 13 transformants in two independent experiments, respectively. In both cases most of the transformants were capable of producing 
conidia and $>90 \%$ of these transformants displayed a yellow phenotype, see Fig. 3A for a representative experiment. To further investigate the nature of the yellow phenotype, we PCR amplified and sequenced the targeted region of $y A$ from a total of twelve randomly selected yellow colonies from the two different experiments. When GE-Oligo1 was used as repair template, ten out of the twelve colonies contained the intended AAC to TAA substitution. The remaining two colonies also contained this substitution, but in each case, the event was accompanied by additional sequence alterations. One of them contained a deletion of a single nucleotide three nucleotides downstream of the TAA mutation; and the other contained a 69 base-pair duplication downstream of the TAA mutation. All transformants obtained with GE-Oligo2 contained the correct AAC to TAA substitution without any additional modifications.

In the experiment described above, the protospacer of yA-sgRNA1 targets the anti-sense strand of $y A$. To investigate whether the efficiency of gene editing was dependent on the strand targeted by Cas9, we exploited that Cas9/yA-sgRNA4 binds to the sense strand of $y A$ to produce a DNA DSB located 384 bp downstream of the DNA DSB produced by Cas9/yA-sgRNA1. This DNA DSB can be repaired by using GE-oligonucleotides GE-Oligo4 and GE-Oligo5, which bind to the anti-sense or sense strands of $y A$, respectively, and which both contain a mutagenic stop-codon (ATC to TGA) centrally in the sequence (Fig. S7B). A co-transformation of NID1 with the pyrG-based CRISPR plasmid pFC920 encoding Cas9/yA-sgRNA4 with GE-Oligo4 or GE-Oligo5 produced 14 and 16 , respectively, healthy transformants of which > $90 \%$ were yellow. Seven yellow transformants obtained with each of the two GE-oligonucleotides were sequenced and this analysis showed that they all contained the expected mutation.

Together our data show that single-stranded GE-oligonucleotides work efficiently as templates for CRISPR mediated gene editing. Moreover, we show that binding of Cas9/sgRNA and binding of GE-oligonucleotides to the coding or non-coding strands do not seem to impact the efficiency of gene editing to any large extent.

\subsection{Single-stranded oligonucleotide mediated CRISPR gene-editing is efficient in other Aspergillus species}

To investigate whether single-stranded oligonucleotide mediated gene editing is applicable in other Aspergillus species, we used a pyrG-based CRISPR plasmid (pFC469) expressing albA-sgRNA, which we have previously used to introduce mutations in $A$. niger with high efficiency (9), and which also matches wA of $A$. oryzae. wA/ albA encodes the polyketide synthase responsible for production of conidia pigment, and fungi where $w A / a l b A$ is deleted produce white conidia. We then designed two 90-mer GE-oligonucleotides, which both contain a mutagenic stop-codon triplet ( $A A C$ to TGA), and which both bind to the sense strands of $A$. niger albA (GEOligo7) and of $A$. oryzae wA (GE-Oligo8) at the position targeted by Cas9/albA-sgRNA.

Ge-Oligo7 and GE-Oligo8 were individually co-transformed with pFC469 into NHEJ deficient strains of $A$. niger and $A$. oryzae, respectively, and plated on solid TM selecting for pFC469. With both fungi we obtained approximately 40 transformants of which approximately $60 \%$ (A. niger) and $80 \%$ (A. oryzae) were white (Fig. 
3B). For each species, eight white transformants were randomly selected and sequenced. In all cases, the desired stop codon was introduced into albA of $A$. niger and in $w A$ of $A$. oryzae at the position directed by GEOligo7 and by GE-Oligo8, respectively. Hence, highly efficient single-stranded oligonucleotide mediated CRISPR gene editing appears to be a common feature of Aspergillus species.

\subsection{Improving gRNA expression for multiplex CRISPR gene editing}

The high CRISPR gene-editing efficiencies in Aspergillus species set the stage for multiplex experiments. However, our current system does not allow for simple delivery of multiple sgRNAs into one strain via a single vector. As an alternative, we envisioned that tRNA-spacer based systems, which have been successfully used for multiplex sgRNA expression in other organisms, including several plants, yeasts, human cells and fruit flies (33-37), could bypass this limitation in filamentous fungi. Specifically, it is exploited that pre-tRNAs contain internal 5'- and 3' splice sites, which are processed by the tRNA splicing machinery where RNase P and RNase Z release mature tRNAs from the larger pre-tRNA transcript (38). In CRISPR experiments, this machinery can be exploited to release sgRNA species. Hence, by constructing genes encoding transcripts containing different sgRNAs species spaced by tRNA entities, multiplexing can be achieved since several different sgRNAs can be liberated from a common transcript by RNase P and RNase Z (Fig. 4).

To build a tRNA based system for gRNA delivery, we first identified a suitable polymerase III promoter as an alternative to the gpdA promoter, which appeared limiting in some experiments, see above. For this purpose, we therefore first constructed genes encoding the $y A$-sgRNA1 sequence fused to the HDV Ribozyme under the control of different polymerase III promoters and the $\operatorname{trpC}$ terminator. Specifically, we tested three polymerase III promoters, the A. fumigatus U6-1 promoter (39), the A. nidulans U6-1 RNA (see M \& M), and the $A$. fumigatus U3 promoter (39) for their ability to induce DNA DSB formation in yA. Hence, pyrG-cas9-AMA1 based plasmids containing yA-sgRNA1 under the control of the promoters Af_U6-1p (pFC899), An_U6-1p (pFC900), and Af_U3p (pFC901) were constructed. pFC899, pFC900, and pFC901 were individually cotransformed into the NHEJ deficient $A$. nidulans strain NID1 along with a circular GT substrate (cGTS-yA-2000) designed for marker free deletion of $y A$. Hence, formation of yellow transformants on solid medium selecting for pFC899, pFC900, and pFC901 serves as an indicator for polymerase III promoter strength. Surprisingly, transformation with pFC899 failed to produce any yellow colonies despite that the U6 promoter is a standard choice for sgRNA expression in many organisms (40-42). Similarly, transformation with pFC900 where yAsgRNA1 expression is controlled by the corresponding promoter from A. nidulans, An_U6-1p, also failed to produce yellow colonies. Encouragingly, with pFC901 almost $100 \%$ of the transformants were yellow (Fig. S8) indicating that the Af_U3p promoter efficiently stimulated yA-sgRNA1 production.

We next examined whether the tRNA splicing machinery could be used to liberate sgRNAs from polymerase III generated transcripts. In this design, one or more sgRNA sequences are individually flanked on both sides by identical glycine tRNA gene sequences to form what we term tRNA splicing-cassettes as the individual sgRNA sequences can be released by endogenous RNase P and RNase Z activities. In a first test of this system, we 
inserted tRNA splicing-cassettes containing a single sgRNA controlled by a U3 promoter and a U3 terminator into an empty CRISPR vector to produce what we term CRISPR-tRNA vectors (for construction see Fig. 5). Specifically we made four pyrG based CRISPR-tRNA vectors pFC902, pFC903, pFC904 and pFC905 that produce yA-sgRNA1, yA-sgRNA2, yA-sgRNA3, and yA-sgRNA4, respectively. We then individually transformed pFC902905 into an NHEJ deficient A. nidulans strain (NID1). On solid medium selecting for the CRISPR-tRNA vectors only few transformants were generated in all cases; and several of these transformants displayed a crippled morphology (Fig. 6). In contrast, control experiments with the corresponding vector, which do not encode a $y A$ specific sgRNA, produced $>70$ healthy transformants. According to TAPE, these experiments indicate that all yA-sgRNA species are efficiently expressed from pFC902-905 resulting in cell death. In agreement with this view, large numbers of transformants with a healthy morphology (20-100) were obtained when pFC902-905 were co-transformed into NID1 with the $y A$ specific GTS, cGTS-yA-2000, indicating that it was efficiently used as a repair template to seal DNA DSBs produced by Cas9/yA-sgRNA1-4. Moreover, the vast majority of the transformants were yellow; and five randomly selected yellow colonies contained the expected $y A$ deletion as judged by diagnostic PCR (Fig. S9). Importantly, the fact that all yA-sgRNA species efficiently stimulated deletion of $y A$ when expressed from pFC902-905 suggests that the concentration of liberated $y A-\operatorname{sgRNA}$ species are higher with the polymerase III/tRNA based system as compared to the corresponding polymerase II/ribozyme based system where yA-sgRNA2 and yA-sgRNA3 do not work efficiently (Compare Fig.1B and Fig. 6). Moreover, the results suggest that TAPE can be used to judge protospacer efficiency in the fungal polymerase III/tRNA based CRISPR system.

\subsection{Introduction of gene deletions by using chimeric repair-oligonucleotides and tRNA mediated multiplex} CRISPR technology

In a first possible use of U3/tRNA mediated multiplexing, we attempted to create gene deletions in an NHEJ deficient strain by using GE-oligonucleotides as repair templates. We envisioned that a specific deletion could be created by introducing two DNA DSBs bordering the sequence to be deleted. In this scenario, a deletion will be formed if the DNA ends up- and downstream of the two DNA DSBs are joined in a repair process guided by a single chimeric GE-oligonucleotide, which matches sequences up- and downstream of the DNA DSBs (Fig. 7A and 7B). We therefore constructed a pyrG-based CRISPR-tRNA vector (pFC1082) containing a yA-sgRNA3 /yAsgRNA4 tRNA splicing-cassette that results in a transcript where yA-sgRNA3 and yA-sgRNA4 are released by

RNase $\mathrm{P}$ and RNase Z. Efficient expression of these genes in A. nidulans is expected to generate two DNA DSBs spaced by 1000 bps in $y A$, and successful repair mediated by a chimeric repair-oligonucleotide will therefore result in a 1000 bps deletion. An NHEJ deficient $A$. nidulans strain (NID1) was co-transformed with the vector and either of two different 90 bps chimeric GE-oligonucleotides; one with a symmetric design where the two homology sections of the GE-oligonucleotide were of the same length, (GE-Oligo11) and, one with an asymmetric design where the 3 -homology section contains $72 \%$ of the bps of the gene-editing oligonucleotide (GE-Oligo12), see Fig. 7B. Encouragingly, in both experiments almost all transformants formed yellow conidia 
demonstrating that $y A$ can be inactivated by this strategy (Fig. 7C and Fig. S10A). In two independent experiments, we noted that the numbers of yellow transformants were approximately two-fold higher when the symmetric GE-oligonucleotide (100 and 101 yellow transformants), rather than the asymmetric GEoligonucleotide (54 and 61 yellow transformants), was used as a repair template. Importantly, diagnostic PCR analyses validated the presence of a $1000 \mathrm{bp}$ yA deletion in eight randomly selected yellow colonies obtained with the symmetric GE-oligonucleotide (Fig. S10B and S10C). To investigate the importance of multiplexing in this gene-deletion method, we attempted to introduce the same $y A$ deletion via single DNA DSBs made by Cas9 guided by gRNA species derived from polymerase III transcripts. Specifically, we individually cotransformed NID1 with pyrG-based CRISPR-tRNA vectors expressing cas9/yA-gRNA1, cas9/yA-gRNA3, or cas9/yA-gRNA4 with the symmetric repair-oligonucleotide. In all three experiments, we did not obtain any colonies despite that we used the same batch of protoplasts as the one used to generate $y A$ deletions in the experiments described above (Fig. 7C). These results indicate that repair of single DNA DSBs located at the borders, or in the center, of the $y A$ sequence via the chimeric repair-oligonucleotide is inefficient.

Next, we investigated whether a deletion mediated by a chimeric repair-oligonucleotide and multiplex CRISPR technology is unique to the $y A$ locus. To this end, we constructed a pyrG-based CRISPR-tRNA vector (pFC994) expressing a $w A-s g R N A 1 / w A-s g R N A 2$ tRNA-based splicing-cassette. Hence, pFC994 produces two new sgRNAs, wA-sgRNA1 and wA-sgRNA2, matching sequences in the up- and downstream region of the PKS gene $w A$. A 90 bp symmetric chimeric repair-oligonucleotide was designed to match the DNA DSBs sites induced by Cas9/wAsgRNA1 and Cas9/WA-sgRNA2 and repair of these breaks stimulated by the repair-oligonucleotide will result in a $7.7 \mathrm{~kb}$ deletion and white conidia. Co-transformation of an NHEJ deficient A. nidulans strain (NID1) with the plasmid pFC994 expressing the WA-sgRNA1/WA-sgRNA2 tRNA-based splicing -cassette and the chimeric GEoligonucleotide resulted in more than 100 colonies of which more than $98 \%$ formed white conidia (Fig. 7D). A PCR analysis (Fig. 7E) of seven randomly selected white colonies demonstrated that all contained the expected deletion of $W A$ (Fig. 7F). These results indicate that this gene-deletion method is locus unspecific.

\subsection{Introduction of multiple gene alterations using repair-oligonucleotides and multiplex CRISPR technology}

In a second possible use of U3/tRNA mediated multiplexing, we attempted to create several genome modifications by using a set of oligonucleotides as repair templates. For example, NID1 contains the mutations $\operatorname{argB2}$, pyrG89, and $n k u A \Delta$ to facilitate genetic engineering. However, these mutations may complicate analyzes of new mutant strains as they potentially impact the phenotype. A simple system to change these mutations into wild-type alleles prior to strain characterization would therefore be desirable. Firstly, we attempted to rescue the single nucleotide deletion and the $\mathrm{G}$ to $\mathrm{A}$ substitution present in $\arg B 2$ and $p y r G 89$, respectively. 90-mer repair-oligonucleotides, GE-Oligo9 and GE-Oligo10, were designed to alter each of the two mutations to wild-type alleles. In the case of pyrG89, the protospacer sequence of the sgRNA did not overlap with the mutation and it was therefore necessary to introduce a silent mutation at a neighboring 
codon to prevent recutting of the corrected pyrG locus. Co-transformation of a NID1 strain with a pyrG-based CRISPR vector expressing $\operatorname{argB2}$-sgRNA1 and with an $\operatorname{argB}$-based CRISPR vector expressing pyrG89-sgRNA1 produced many transformants on solid $\mathrm{TM}+\mathrm{Arg}$ or $\mathrm{TM}+\mathrm{Ura}+\mathrm{Uri}$ indicating that the relevant mutations have been cured in each of the two cases. Interestingly, in corresponding control experiments where GEoligonucleotides were transformed into NID1 along with an empty CRISPR vector, a few transformants could still be obtained on solid TM+Arg or TM+Ura+Uri (data not shown). Next, we constructed an hph-based CRISPR-tRNA plasmid containing an argB2-sgRNA1/pyrG89-sgRNA1 tRNA-based splicing-cassette and cotransformed this vector into NID1 together with GE-Oligo9 and GE-Oligo10. This experiment produced many transformants on solid TM strongly indicating that both mutations have been simultaneously cured. In this case, the corresponding control experiment did not produce any transformants on solid TM (data not shown). Finally, we used TAPE to identify an sgRNA that efficiently targets Cas9 to $n k u A \triangle$ (Fig. S11) to set the stage for rescuing this allele.

Encouraged by the results presented above, we next attempted to rescue all three NID1 mutations simultaneously. For this purpose, we created an $h p h$-based CRISPR-tRNA vector expressing an argB2sgRNA1/pyrG-sgRNA1/nkuAL-sgRNA1 tRNA-based splicing-cassette and co-transformed this vector into NID1 together with the $\arg B$ and $p y r G$ repair oligonucleotides as well with a circular GT substrate containing a functional $n k u A$ gene. Two transformation experiments were performed and more than 20 transformants were obtained in each experiment using selection on solid TM. Ten transformants were randomly selected and tested by diagnostic PCR for restoration of $n k u A$ (Fig. S12). All ten transformants contained clean insertions as no trace of the $n k u A \Delta$ allele could be observed in any of the analyses. Next, the $\arg B$ and pyrG loci of the 10 transformants were amplified by PCR and sequenced. In all ten transformants, the $\arg B$ and $p y r G$ alleles were repaired correctly. However, in one of these transformants, the silent mutation intended to accompany pyrG restoration was not incorporated. Altogether, in nine of the ten transformants, all three repair events were preformed correctly showing that U3/tRNA mediated multiplexing can be efficiently used to introduce multiple gene alterations at the same time.

\section{Discussion}

In eukaryotes, two major pathways, NHEJ and HR, are required for efficient repair of DNA DSBs and in this report we present data demonstrating that both NHEJ and HR DNA repair pathways engage in repair of Cas9 induced DNA DSBs in Aspergilli. Hence, efficient CRISPR vectors readily stimulate introduction of mutations into a specific locus targeted by Cas9/sgRNA in NHEJ proficient, but not in deficient NHEJ strains. Moreover, we find that efficient CRISPR vectors cause massive cell death in NHEJ deficient strains. This lethality can be cured by providing DNA that serves as a repair template in HR repair of Cas9/sgRNA induced DNA DSBs. Here we have exploited these facts to improve CRISPR-based gene editing in Aspergilli. 
Like with S. cerevisiae, we exploited CRISPR plasmid induced lethality to identify protospacers that efficiently targets Cas9 to specific sequence motifs and we call this method TAPE. Here we show that TAPE can be used to identify protospacers that efficiently induce DNA DSBs for efficient CRISPR-mediated gene manipulations. It has recently been shown that catalytically dead Cas9 (dCas9) variants can be exploited as synthetic transcription (43-45); and with S. cerevisiae, we have recently used TAPE to identify protospacers that efficiently target dCas9 to specific promoter sequences to successfully regulate metabolism in predicted manners (32). We are currently implementing the same system in Aspergilli with the aim of regulating genes in secondary metabolite clusters since we believe it can serve as a useful tool in the discovery of new SMs and in the dissection of SM pathways.

In our original setup for fungal CRISPR/Cas9-mediated gene editing, we used a polymerase II promoter, PgpdA, to express the sgRNA. As a consequence, the mature sgRNA needs to be liberated from a larger transcript. The fact that the $\mathrm{HH}$ - and HDV ribozymes catalyze formation of only a single RNA break complicates construction of polycistronic transcripts, from which several different sgRNA species can be released. We bypassed this limitation by exploiting that evolutionary highly conserved RNase $\mathrm{P}$ and RNase Z release tRNAs from a larger transcript by making incisions up- and downstream of the tRNA, respectively. Hence, different sgRNAs can be released from a common transcript if all individual sgRNA species are flanked by tRNA sequences (see Fig. 4). Moreover, we identified a functional polymerase III promoter Af_P-U3 to substitute for PgpdA. By designing a vector set that allows for simple construction of synthetic genes where these two features are combined, we have created a very efficient setup for fungal gene editing and demonstrated that three genetic events can be simultaneously changed in a single round of transformation with very high efficiency. It will be interesting to determine the upper limit of how many genetic alterations can by introduced via expression of a single tRNAbased splicing-cassette. In our setup, we used identical glycine tRNA species as spacers, and splicing cassettes containing many sgRNAs may be unstable due to direct-repeat recombination. To reduce this problem, different tRNAs may need to be implemented in our system. Importantly, we envision that powerful multiplex systems like the one presented here will greatly reduce the workload for complex genetic engineering of fungi.

With the aim of optimizing Cas9/sgRNA mediated gene targeting, we analyzed different GTSs for genetargeting efficiencies. These experiments showed that circular designs appear more efficient than the corresponding linear designs. This may be due to the fact that circular GTSs cannot directly enter the NHEJ pathway as no free ends are available for binding NHEJ proteins and this may favor repair by HR. In addition, the lack of free ends may also protect circular GTS against exonucleases to extend the time where it may serve as a repair template. For construction of a limited number of mutations by CRISPR we therefore recommend the use of circular GTSs. However, construction of plasmid based GTSs is laborious and constitutes a major bottleneck towards the creation of defined genome-wide deletion libraries and it would therefore be highly advantageous if efficient gene targeting could be achieved via a simpler and less expensive GTS type. With this in mind, we investigated whether single-stranded oligonucleotides could serve as templates for gene-editing. In the yeast S. cerevisiae, I-Scel and Cas9 induced DNA DSB have been efficiently used to stimulate gene editing 
with oligonucleotides $(46,18)$. In both cases, gene editing with double-stranded oligonucleotides was about 310 times more efficient than with single-stranded oligonucleotides. In our setup for gene editing in Aspergilli, where we select for the CRISPR plasmid, but not the new mutation, we obtain gene-editing efficiencies approaching $100 \%$ with single-stranded GE-oligo nucleotides indicating that there is no need for using doublestranded oligonucleotides for gene-editing experiments. The observation that gene editing with singlestranded oligonucleotides appear more efficient than linear GTSs in gene-targeting experiments may likely be due to the much higher concentrations of single-stranded oligonucleotides relative to linear plasmid derived GTSs in CRISPR experiments. In our experiments we have typically used more than 2000-fold higher concentrations of single-stranded oligonucleotides as compared to linear GTSs. Lastly, since efficient genetargeting with linear GTSs with short targeting sequences have been reported for fungi belonging to other genera $(10,13,42)$, we therefore envision that the use of single-stranded oligonucleotides in CRISPR mediated gene editing may be of broad use in the fungal kingdom.

In a previous (9), as well as in the present paper, we find that our CRISPR mediated gene targeting tools work well in a number of species. However, we have also observed species where our CRISPR technology worked with low efficiency. In the present work, we find that gene targeting into the $y A$ locus in $A$. nidulans appears much more efficient with pyrG based CRISPR vectors as compared to the levels obtained with argB based CRISPR vectors. Our mRFP expression analyses indicate that this difference may be explained by a combination of higher and more uniform mRFP expression levels in strains that contains the pyrG based CRISPR vector. As a result, we believe that strains transformed with this plasmid type contain a higher number of nuclei with sufficient concentrations of Cas9/sgRNA complexes to support efficient gene targeting. For species with low CRISPR mediated gene-targeting efficiencies it may therefore be useful to determine the strength of the relevant promoters to ensure that sufficient levels of Cas 9 and sgRNA are produced. Similarly, if AMA1 based vectors are used for cas9 and $s g R N A$ gene expression, it may be advisable to determine plasmid stability.

In summary we have expanded the toolbox for fungal CRISPR technology by developing TAPE to assess protospacer efficiency, by identifying a useful polymerase III promoter for sgRNA production and by developing CRIPSR-tRNA vectors that set the stage for multiplex experiments as multiple different sgRNA can be spliced out of a common transcript. Importantly, we also show that single-stranded oligonucleotides can be directly used for marker-free CRISPR gene editing to efficiently introduce specific point mutations and gene deletions, hence, eliminating the need for construction of complex gene-targeting substrates. Together we envision that these tools will greatly facilitate genetic engineering of fungi including even the possibility of performing high-throughput experiments.

\section{Appendix A. Supplementary material}

Supplementary data are available at FG\&B online. 


\section{Acknowledgements}

We thank Gijs Verkleij and Evan Chaberski for technical assistance, Maria L. Nielsen and Ferdinand H. Kirchner for helpful scientific discussions; and Katherina G. Vanegas and Line H. Kristensen for carefully reading this manuscript. This work was financially supported by Novozymes A/S and by grant 11-116803 from The Danish Council for Strategic Research.

\section{References}

1. Fleißner,A. and Dersch,P. (2010) Expression and export: recombinant protein production systems for Aspergillus. Appl. Microbiol. Biotechnol., 87, 1255-1270.

2. Moretti, a., Susca,A., Mulé,G., Logrieco,A.F. and Proctor,R.H. (2013) Molecular biodiversity of mycotoxigenic fungi that threaten food safety. Int. J. Food Microbiol., 167, 57-66.

3. Newman,D.J. and Cragg,G.M. (2016) Natural Products as Sources of New Drugs from 1981 to 2014. J. Nat. Prod., 79, 629-661.

4. Hoffmeister,D. and Keller,N.P. (2007) Natural products of filamentous fungi: enzymes, genes, and their regulation. Nat. Prod. Rep., 24, 393-416.

5. Wu,F., Groopman,J.D. and Pestka,J.J. (2014) Public Health Impacts of Foodborne Mycotoxins. Annu. Rev. Food Sci. Technol., 5, 351-372.

6. de Vries,R.P., Riley,R., Wiebenga,A., Aguilar-Osorio,G., Amillis,S., Uchima,C.A., Anderluh,G., Asadollahi,M., Askin,M., Barry,K., et al. (2017) Comparative genomics reveals high biological diversity and specific adaptations in the industrially and medically important fungal genus Aspergillus. Genome Biol., 18, 28.

7. Nielsen,J.C., Grijseels,S., Prigent,S., Ji,B., Dainat,J., Nielsen,K.F., Frisvad,J.C., Workman,M. and Nielsen,J. (2017) Global analysis of biosynthetic gene clusters reveals vast potential of secondary metabolite production in Penicillium species. Nat. Microbiol., 10.1038/nmicrobiol.2017.44.

8. Anyaogu,D.C. and Mortensen,U.H. (2015) Heterologous production of fungal secondary metabolites in Aspergilli. Front. Microbiol., 6, 1-6.

9. Nødvig,C.S., Nielsen,J.B., Kogle,M.E. and Mortensen,U.H. (2015) A CRISPR-Cas9 System for Genetic Engineering of Filamentous Fungi. PLoS One, 10, e0133085.

10. Liu,R., Chen,L., Jiang,Y., Zhou,Z. and Zou,G. (2015) Efficient genome editing in filamentous fungus Trichoderma reesei using the CRISPR/Cas9 system. Cell Discov., 1, 15007. 
11. Arazoe,T., Miyoshi,K., Yamato,T., Ogawa,T., Ohsato,S., Arie,T. and Kuwata,S. (2015) Tailor-made CRISPR/Cas system for highly efficient targeted gene replacement in the rice blast fungus. Biotechnol. Bioeng., 112, 2543-2549.

12. Matsu-ura,T., Baek,M., Kwon,J. and Hong,C. (2015) Efficient gene editing in Neurospora crassa with CRISPR technology. Fungal Biol. Biotechnol., 2, 4.

13. Pohl,C., Kiel,J.A.K.., Driessen,A.J.M., Bovenberg,R.A.L. and Nygård,Y. (2016) CRISPR/Cas9 based genome editing of Penicillium chrysogenum. ACS Synth. Biol., 10.1021/acssynbio.6b00082.

14. Krappmann,S. (2016) CRISPR-Cas9, the new kid on the block of fungal molecular biology. Med. Mycol., 10.1093/mmy/myw097.

15. Jinek,M., East,A., Cheng,A., Lin,S., Ma,E. and Doudna,J. (2013) RNA-programmed genome editing in human cells. Elife, 2, e00471.

16. Ran,F.A., Hsu,P.D., Wright,J., Agarwala,V., Scott,D. a and Zhang,F. (2013) Genome engineering using the CRISPR-Cas9 system. Nat. Protoc., 8, 2281-2308.

17. Mali,P., Yang,L., Esvelt,K.M., Aach,J., Guell,M., DiCarlo,J.E., Norville,J.E. and Church,G.M. (2013) RNAGuided Human Genome Engineering via Cas9. Science (80-. )., 339, 823-826.

18. Dicarlo,J.E., Norville,J.E., Mali,P., Rios,X., Aach,J. and Church,G.M. (2013) Genome engineering in Saccharomyces cerevisiae using CRISPR-Cas systems. Nucleic Acids Res., 41, 4336-4343.

19. Doudna,J.A. and Charpentier,E. (2014) The new frontier of genome engineering with CRISPR-Cas9. Science (80-. )., 346, 1258096-1258096.

20. Nielsen,M.L., Isbrandt,T., Rasmussen,K.B., Thrane,U., Hoof,J.B., Larsen,T.O. and Mortensen,U.H. (2017) Genes Linked to Production of Secondary Metabolites in Talaromyces atroroseus Revealed Using CRISPRCas9. PLoS One, 12, e0169712.

21. Kaminskyj,S.G.W. (2001) Fundamentals of growoth, storage, genetics and microscopy of Aspergillus nidulans. Fungal Genet. Newsl.

22. Cove,D.J. (1966) The induction and repression of nitrate reductase in the fungus Aspergillus nidulans. Biochim. Biophys. Acta, 113, 51-56.

23. Arnaud,M.B., Cerqueira,G.C., Inglis,D.O., Skrzypek,M.S., Binkley,J., Chibucos,M.C., Crabtree,J., Howarth,C., Orvis,J., Shah,P., et al. (2012) The Aspergillus Genome Database (AspGD): Recent developments in comprehensive multispecies curation, comparative genomics and community resources. Nucleic Acids Res., 40, D653-D659. 
24. Stajich,J.E., Harris,T., Brunk,B.P., Brestelli,J., Fischer,S., Harb,O.S., Kissinger,J.C., Li,W., Nayak,V., Pinney,D.F., et al. (2012) FungiDB: An integrated functional genomics database for fungi. Nucleic Acids Res., 40, 675681.

25. Nour-Eldin,H.H., Geu-Flores,F. and Halkier,B.A. (2010) USER cloning and USER fusion: the ideal cloning techniques for small and big laboratories. Methods Mol. Biol., 643, 185-200.

26. Hansen,B.G., Salomonsen,B., Nielsen,M.T., Nielsen,J.B., Hansen,N.B., Nielsen,K.F., Regueira,T.B., Nielsen,J., Patil,K.R. and Mortensen,U.H. (2011) Versatile enzyme expression and characterization system for Aspergillus nidulans, with the Penicillium brevicompactum polyketide synthase gene from the mycophenolic acid gene cluster as a test case. Appl. Environ. Microbiol., 77, 3044-3051.

27. Nørholm,M.H.H. (2010) A mutant Pfu DNA polymerase designed for advanced uracil-excision DNA engineering. BMC Biotechnol., 10, 21.

28. Nielsen,M.L., Albertsen,L., Lettier,G., Nielsen,J.B. and Mortensen,U.H. (2006) Efficient PCR-based gene targeting with a recyclable marker for Aspergillus nidulans. Fungal Genet. Biol. , 43, 54-64.

29. Plate,I., Hallwyl,S.C.L., Shi,I., Krejci,L., Müller,C., Albertsen,L., Sung,P. and Mortensen,U.H. (2008) Interaction with RPA is necessary for Rad52 repair center formation and for its mediator activity. J. Biol. Chem., 283, 29077-29085.

30. Clutterbuck ,A.J. (1972) Absence of Laccase from Yellow-spored Mutants of Aspergillus nidulans. J. Gen. Microbiol., 70, 423-435.

31. Gao,Y. and Zhao,Y. (2014) Self-processing of ribozyme-flanked RNAs into guide RNAs in vitro and in vivo for CRISPR-mediated genome editing. J. Integr. Plant Biol., 56, 343-349.

32. Vanegas,K.G., Lehka,B.J. and Mortensen,U.H. (2017) SWITCH: a dynamic CRISPR tool for genome engineering and metabolic pathway control for cell factory construction in Saccharomyces cerevisiae. Microb. Cell Fact., 16, 25.

33. Xie,K., Minkenberg,B. and Yang,Y. (2015) Boosting CRISPR/Cas9 multiplex editing capability with the endogenous tRNA-processing system. Proc. Natl. Acad. Sci., 112, 3570-3575.

34. Port,F. and Bullock,S.L. (2016) Augmenting CRISPR applications in Drosophila with tRNA-flanked sgRNAs. Nat. Methods, 4, 1-5.

35. Qi,W., Zhu,T., Tian,Z., Li,C., Zhang,W. and Song,R. (2016) High-efficiency \{CRISPR/Cas9\} multiplex gene editing using the glycine \{tRNA-processing system-based strategy in maize. Bmc Biotechnol, 16, 58.

36. Schwartz,C.M., Hussain,M.S., Blenner,M. and Wheeldon,I. (2015) Synthetic RNA polymerase III promoters facilitate high efficiency CRISPR-Cas9 mediated genome editing in Yarrowia lipolytica. ACS Synth. Biol., 
10.1021/acssynbio.5b00162.

37. Dong,F., Xie,K., Chen,Y., Yang,Y. and Mao,Y. (2017) Polycistronic tRNA and CRISPR guide-RNA enables highly efficient multiplexed genome engineering in human cells. Biochem. Biophys. Res. Commun., 482, 889895.

38. Phizicky,E.M. and Hopper,A.K. (2010) tRNA biology charges to the front. Genes Dev., 24, 1832-1860.

39. Jöchl,C., Rederstorff,M., Hertel,J., Stadler,P.F., Hofacker,I.I., Schrettl,M., Haas,H. and Hüttenhofer,A. (2008) Small ncRNA transcriptome analysis from Aspergillus fumigatus suggests a novel mechanism for regulation of protein synthesis. Nucleic Acids Res., 36, 2677-2689.

40. Sander,J.D. and Joung,J.K. (2014) CRISPR-Cas systems for editing, regulating and targeting genomes. Nat. Biotechnol., 32, 347-355.

41. Ma,H., Wu,Y., Dang,Y., Choi,J.-G., Zhang,J. and Wu,H. (2014) Pol III Promoters to Express Small RNAs: Delineation of Transcription Initiation. Mol. Ther. Acids, 3, e161.

42. Zhang,C., Meng,X., Wei,X. and Lu,L. (2015) Highly efficient CRISPR mutagenesis by microhomologymediated end joining in Aspergillus fumigatus. Fungal Genet. Biol., 86, 47-57.

43. Jinek,M., Chylinski,K., Fonfara,I., Hauer,M., Doudna,J.A. and Charpentier,E. (2012) A Programmable DualRNA-Guided DNA Endonuclease in Adaptive Bacterial Immunity. Science (80-. )., 337, 816-821.

44. Gilbert,L. a, Larson,M.H., Morsut,L., Liu,Z., Brar,G. a, Torres,S.E., Stern-Ginossar,N., Brandman,O., Whitehead,E.H., Doudna,J. a, et al. (2013) CRISPR-mediated modular RNA-guided regulation of transcription in eukaryotes. Cell, 154, 442-51.

45. Vora,S., Tuttle,M., Cheng,J. and Church,G. (2016) Next stop for the CRISPR revolution: RNA-guided epigenetic regulators. FEBS J., 283, 3181-3193.

46. Storici,F., Durham,C.L., Gordenin,D. a and Resnick,M. a (2003) Chromosomal site-specific double-strand breaks are efficiently targeted for repair by oligonucleotides in yeast. Proc. Natl. Acad. Sci. U. S. A., 100, 14994-14999.

\section{TABLE AND FIGURES LEGENDS}

Table 1. Strains used in this study.

Table 2. Gene-targeting efficiency depends on homology length of the gene-targeting substrate. 
Fig. 1. TAPE predicts efficient sgRNAs for robust gene targeting. Three experimental sets of transformation results obtained by transforming $A$. nidulans with CRISPR plasmids encoding Cas 9 and either no sgRNA (control plasmid), yA-sgRNA1, yA-sgRNA2, yA-sgRNA3, or yA-sgRNA4 as indicated. Experimental set 1 analyzed transformation of NHEJ proficient strain (NID1), and sets 2 and 3 analyzed NHEJ deficient strain (NID5) in the absence and presence of a circular gene-targeting substrate (GTS) as indicated. (A) The approximate binding sites in yA of sgRNAs yA-sgRNA1-4. The arrowheads indicate the orientation of the protospacers relative to coding (pointing to the right) and non-coding (pointing to the left) strands. (B) Representative plates obtained from experimental sets 1-3. (C) Numbers of conidiating transformants in \% relative to the number obtained with the empty control CRISPR plasmid are shown for each of experimental sets 1-3. A * above columns indicates that the relative numbers of transformants obtained with a CRISPR plasmid expressing a given sgRNA are significantly different $(p \leq 0.05)$ from the number obtained with the empty control plasmid in that experimental set. ** above columns indicates that relative numbers of transformants obtained in set1 are significantly different $(p \leq 0.05)$ from the corresponding numbers obtained in set $2 . A^{* * *}$ above columns indicates that relative numbers of transformants obtained in set 2 are significantly different $(p \leq 0.05)$ from the corresponding numbers in set 3 .

Fig. 2. Protein production from AMA1 plasmids shows dependency on the selective marker. Conidia from NID1 transformants containing AMA1 based plasmids expressing an mRFP gene under the control of the tef1 promoter. Images show analyses of strains containing plasmids selected by either the pyrG or the $\arg B$ marker as indicated. For plasmid details, see Table S4. Images in the row labeled DIC are made by differential interference contrast microscopy using 1000X magnification. Images in the row labeled mRFP are fluorescent microscopy images captured of the same cells using $50 \mathrm{~ms}$ exposure times.

Fig. 3. Efficient single-stranded oligonucleotide mediated CRISPR gene-editing in Aspergilli. (A) Cotransformations of NHEJ deficient $A$. nidulans NID1 strain with the pyrG-based CRISPR vector pFC897 encoding YA-sgRNA1 and a gene-editing (GE-) oligonucleotide targeting yA, (top) GE-Oligo1 or (bottom) GE-Oligo2. (B) Co-transformations of NHEJ deficient A. niger (NIG96) and A. oryzae (PFJo218) strains with GE-oligonucleotides targeting albA (GE-Oligo7) and wA (GE-Oligo8), respectively, and (top) with either an empty CRISPR plasmid (pFC330) or (bottom) or with the CRISPR plasmid encoding the sgRNA targeting albA and $w A$ (pFC469).

Fig. 4. tRNA processing liberates of multiple sgRNA species from a single pre-RNA transcript. Individual sgRNA species are flanked by tRNA sequences. Dotted lines and "Pacmen" indicate 
cleavage sites for RNase $\mathrm{P}$ and RNase $\mathrm{Z}$ in the pre-RNA transcript. The different colors of the protospacer moiety of individual sgRNAs indicate that they contain different sequences.

Fig. 5. Construction of CRISPR-tRNA vectors for tRNA in vivo catalyzed sgRNA release. (A) A finalized CRISPR-tRNA vector (we use pFC902, see Fig. S2) serves as template for PCR reactions generating new sgRNA genes for new CRISPR-tRNA vectors. Two vector construction tracks are shown in the figure. For both tracks, all primers contain tails designed for USER fusion as conceptualized in Fig. S1. All PCR fragments are treated with USER enzyme, mixed with an empty CRISPR vector fragment where the USER-cassette has been opened with Pacl and Nt.BbvCl (9), and transformed into E. coli for in vivo vector assembly by USER fusion. Four different markers (pyrG (pFC330), $\arg B(\mathrm{pFC} 331)$, $h p h(\mathrm{pFC} 332)$, and ble (pFC333)) are currently available as empty CRISPR vectors (see (9) and Supplementary Table S2).The "Single sgRNA Splicing-Cassette" track is used to construct a vector that produces a single sgRNA. In one PCR reaction, a fragment (labeled AB in the diagram) containing the PAf_U3 promoter followed by a tRNA gene is amplified by primers $a$ and $b$. In another PCR gene reaction, a fragment, CD, containing an sgRNA, a tRNA gene and a TAf_U3 terminator is generated by using primers $\mathrm{c}$ and $\mathrm{d}$. Importantly, the tails of primers $\mathrm{b}$ and $\mathrm{c}$ contain overlapping parts of the desired protospacer sequence, and the full sgRNA is formed when fragments $A B$ and $C D$ are fused via these primer tails. Note that matching primer tails for USER fusion are indicated by identical colors. The "Multiple sgRNA Splicing-Cassette" track is used to construct a vector that produces several different sgRNAs; the example shows how to make a vector that produces three different sgRNAs. Like in the "Single sgRNA Splicing-Cassette" track, a fragment, AB, containing the PAf_U3 promoter followed by a tRNA gene is amplified by primers $a$ and $b$ in one PCR reaction. In two individual PCR reactions (the number depends on how many different sgRNAs a given vector should produce) two different sgRNA-tRNA fusion fragments, CF and EH, are produced in reactions containing primers $\mathrm{c}$ and $\mathrm{f}$, and $\mathrm{e}$ and $\mathrm{h}$, respectively. The last PCR fragment, GD, which contains the last sgRNA followed by a tRNA gene and a TAf_U3 terminator, is amplified by primers $g$ and $d$. As in the "Single sgRNA Splicing-Cassette" track, all primers contain tails designed for USER fusion, and the sgRNA sequences, including the full protospacer sequences, are established via the primer tail mediated fusions. In the final CRISPR-tRNA vectors, all sgRNA encoding sequences are positioned between identical tRNA (Gly) genes. (B) Illustration showing the USER fusion of a 2-sgRNA SplicingCassette through two different protospacers at nucleotide level of resolution (see Fig. S1 for concept). Red " $U$ "s represent uracil residues. The desired protospacer sequence is defined by the sequences in the primer tails represented by " $\mathrm{n}$ " $\mathrm{s}$ and " $\mathrm{N}$ " $\mathrm{s}$, hence, indicating that any sequence can 
be chosen. Importantly, for each protospacer, the sequence defined by " $n$ "s between $U$ and $A$, and, the sequence defined by " $\mathrm{N}$ "s between $\mathrm{A}$ and $\mathrm{U}$, need to be complementary to allow for USER fusion.

Fig. 6. sgRNAs liberated from RNA polymerase III transcripts based on intracellular ribozyme processed tRNAs efficiently stimulate CRISPR based gene targeting. Transformation of NHEJ deficient NID1 strains with tRNA-CRISPR plasmids encoding Cas9 and either no sgRNA, yA-sgRNA1, yA-sgRNA2, yA-sgRNA3, and yA-sgRNA4 in the absence or presence of a circular GTS containing 2000 bps of homologous up- and down-stream yA targeting sequences (cGTS-yA-2000). In all cases, sgRNA genes are controlled by the U3 promoter and mature sgRNAs are liberated by glycine tRNA sequences embedded in the same pre-RNA transcript.

Fig. 7. Gene-editing oligonucleotide mediated gene deletion in A. nidulans by CRISPR. (A) Positions of sgRNA target sequences (thin black lines) and of targeting sequences complementary to the chimeric gene-editing (GE-) oligonucleotide (indicated by purple and blue lines) in A. nidulans $y A$. Drawing is not to scale. (B) Designs of chimeric GE-oligonucleotides for $y A$ gene deletion. Purple and blue sections bind to the complementary (purple and blue) sections of the $y A$ gene as presented in panel A. (C) Transformation of $A$. nidulans NHEJ deficient NID1 with CRISPR-tRNA plasmids encoding Cas9 and sgRNAs as indicated. GE-Oligo11 is used as repair template. (D) C-transformation of NID1 with a pyrG based CRISPR-tRNA plasmid encoding Cas9 and two sgRNAs targeting each end of the $w A$ locus in and a chimeric GE-oligonucleotide matching sequences up- and down-stream of the expected upand downstream DNA DSBs in the albA gene, see text for details. (E) Drawing of the wA locus. Blue arrows indicate position of Cas9-sgRNA target sites. Black arrows indicate primer positions for diagnostic PCR analyses. PCR fragment sizes expected from $w A$ and $w A \Delta$ strains are indicated. (F) Analysis of diagnostic PCR reactions by agarose gel electrophoresis. Two independent PCR reactions were performed for each of the seven transformants analyzed. $C$ represents the analysis of the untransformed control strain, NID1. 
A

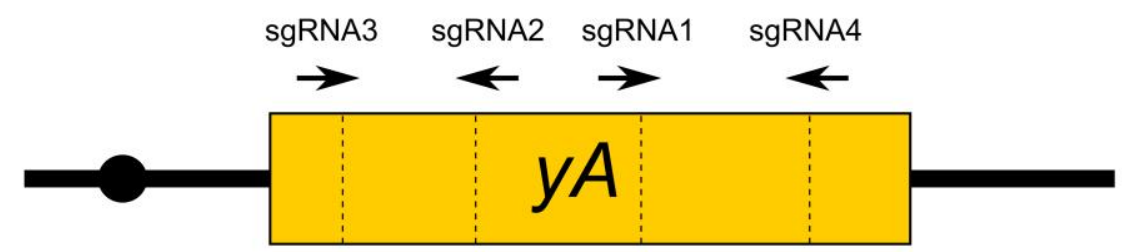

B

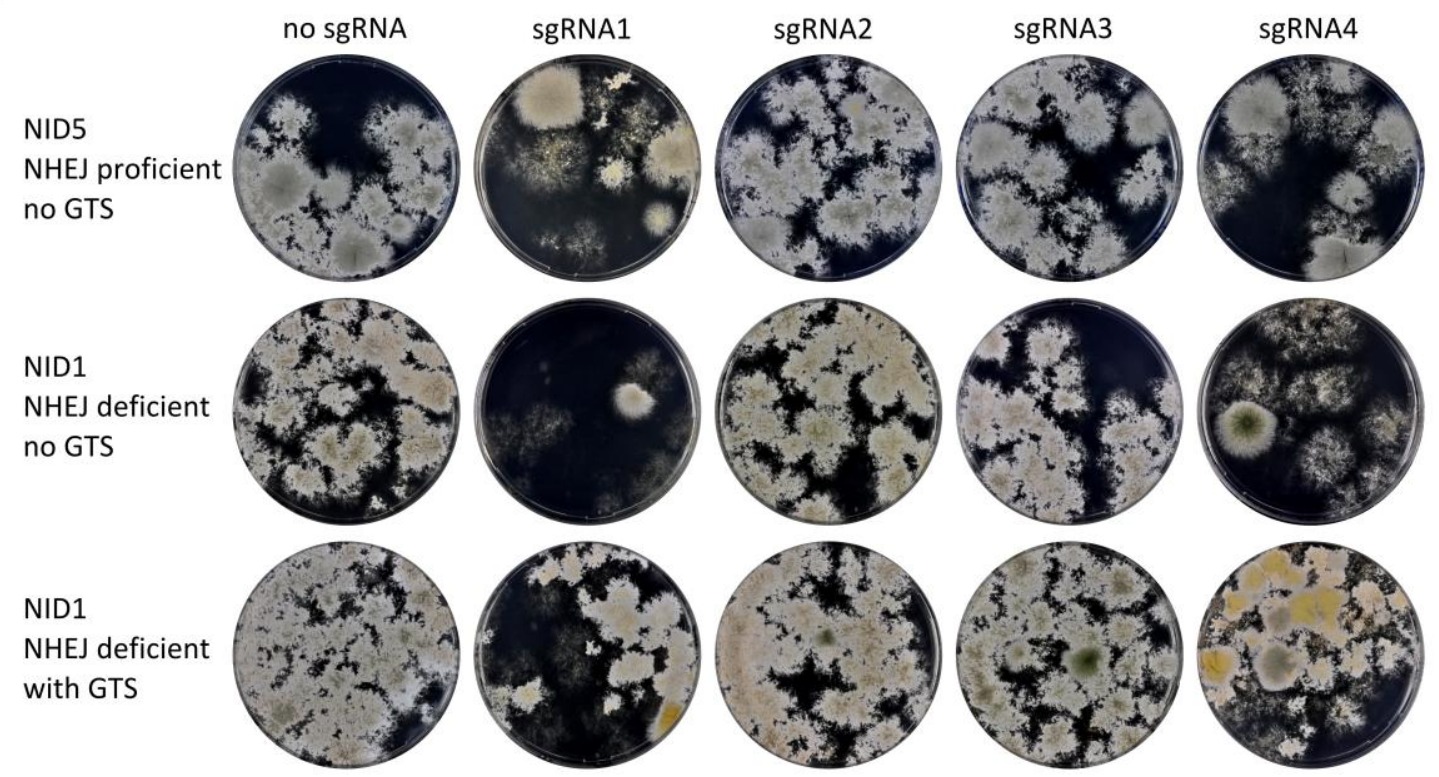

C

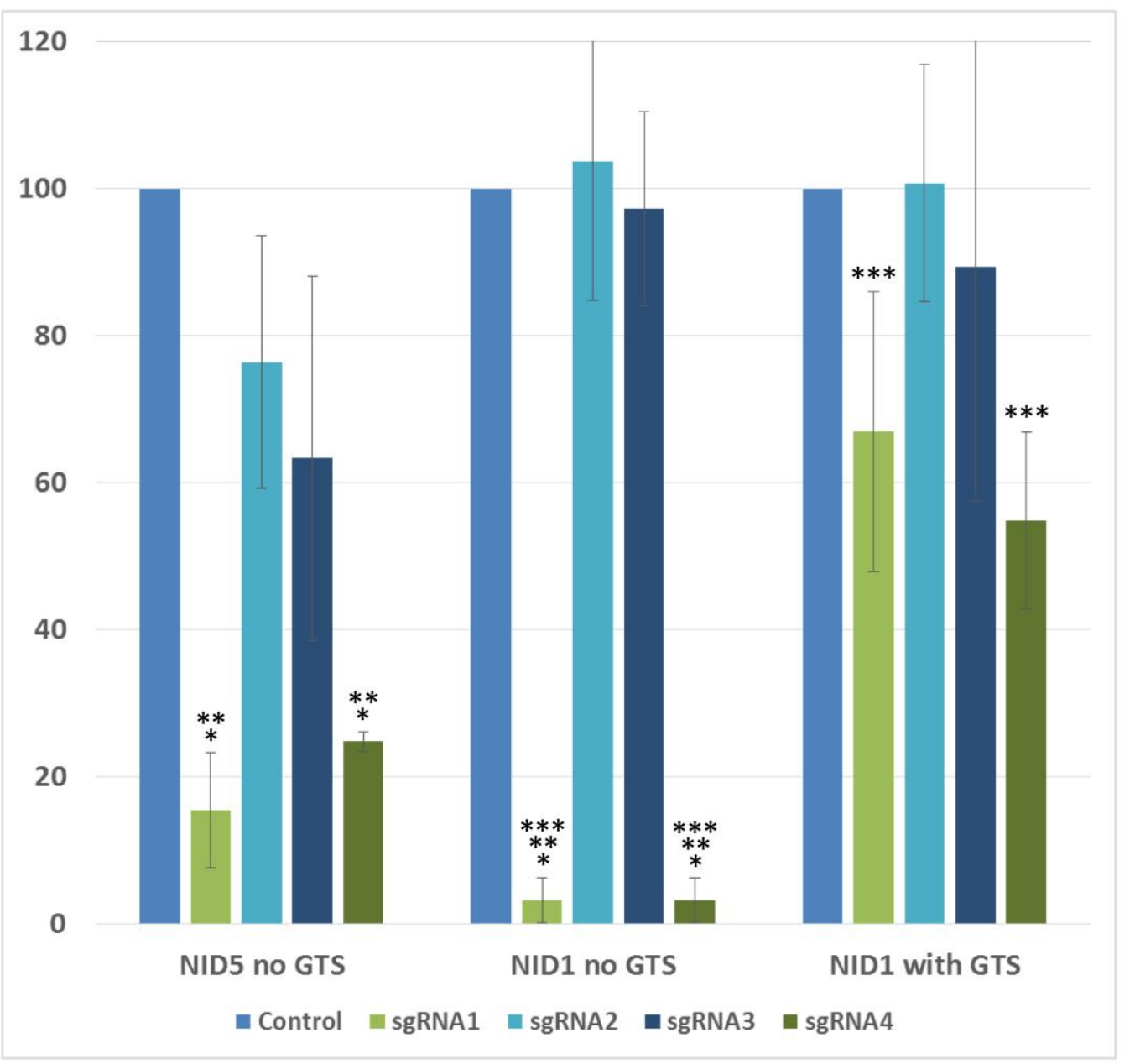


pyrG

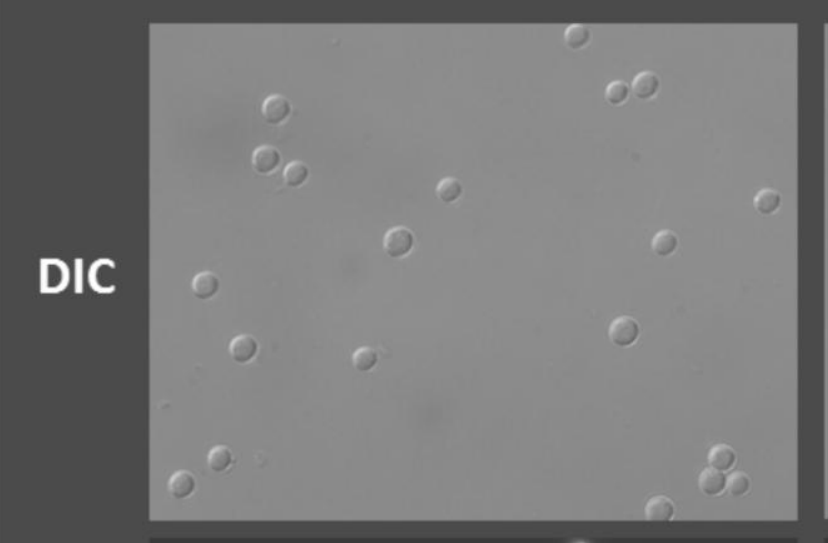

a

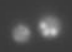

MRFP

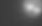

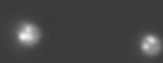

$\varphi^{8}$
9

9.

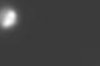

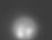

s

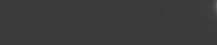

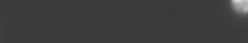

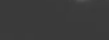

8

argB

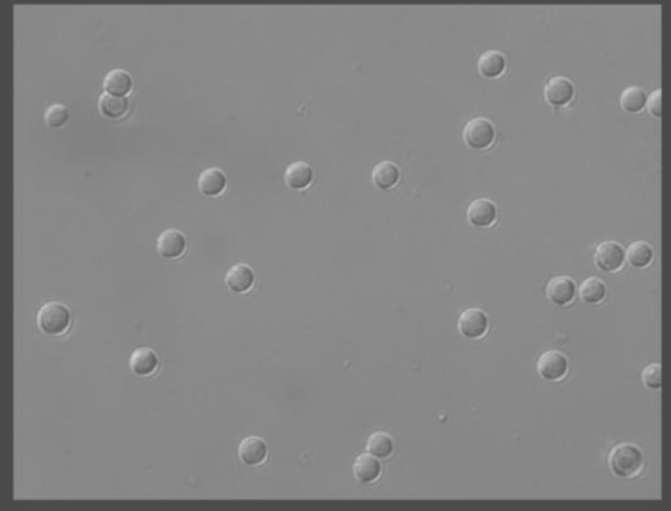

(3)

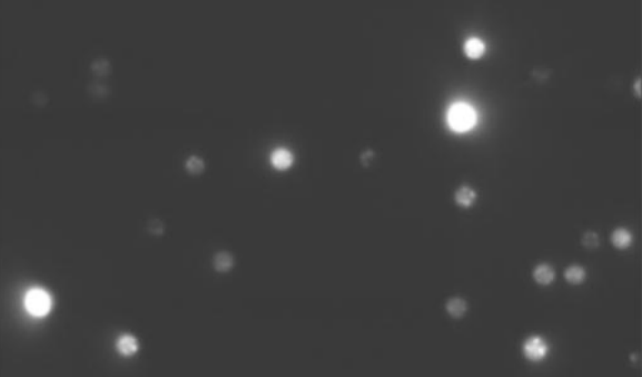

e 

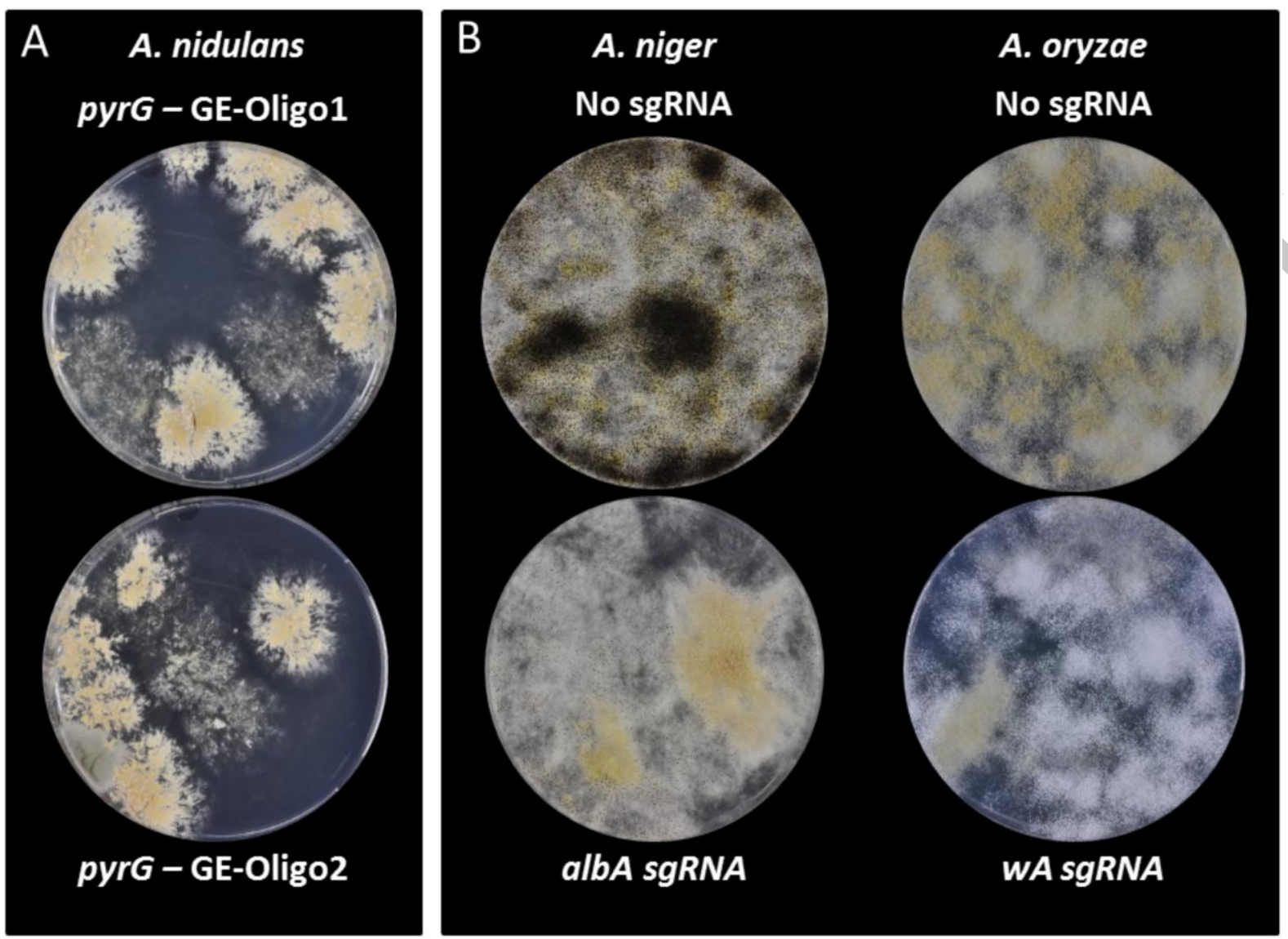


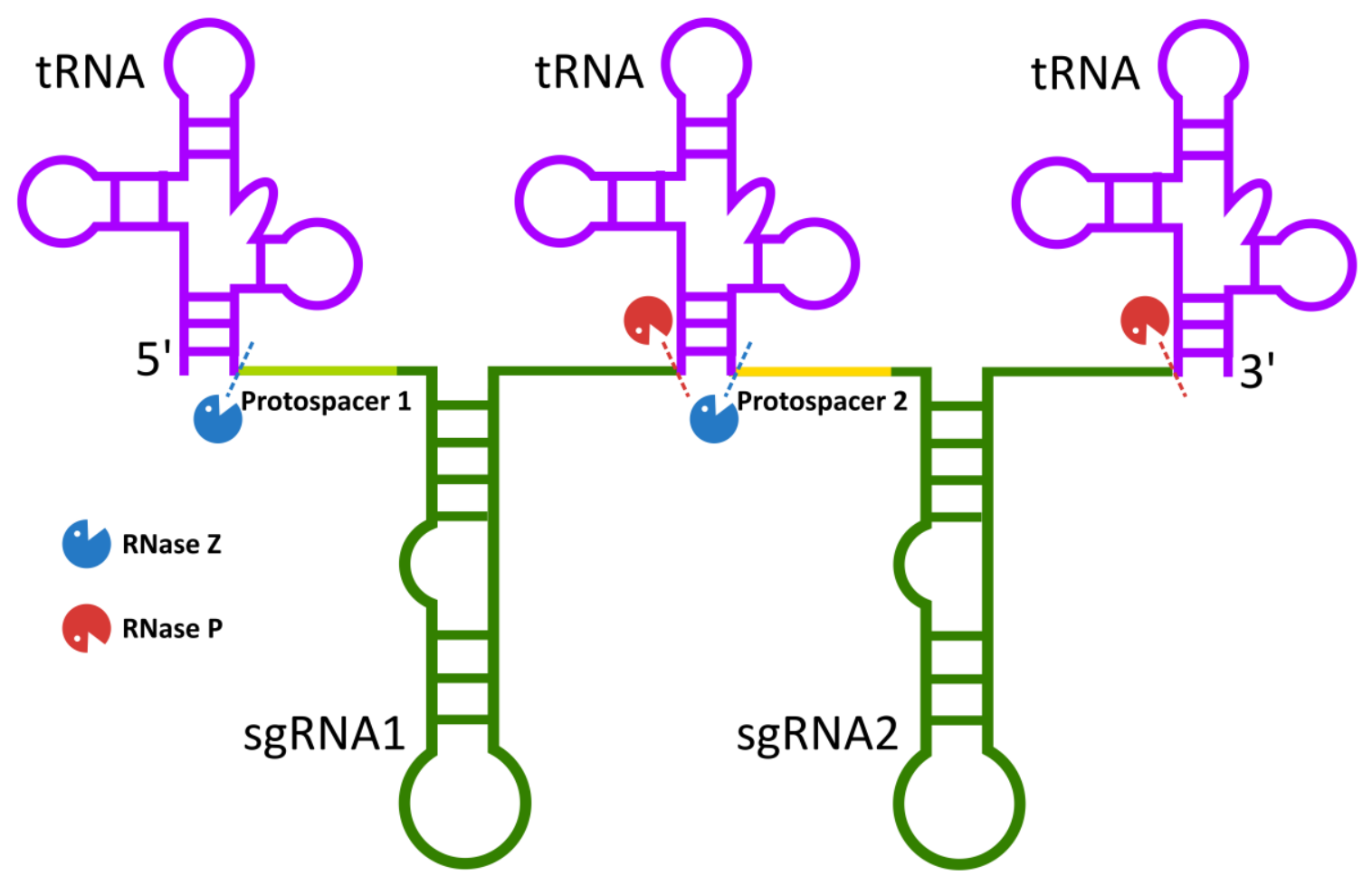


A

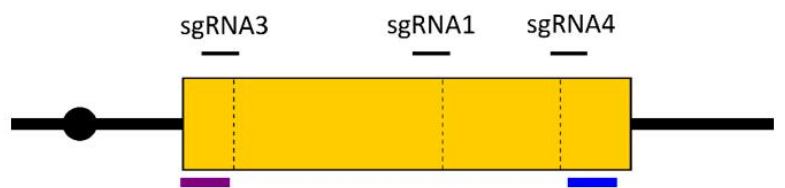

B

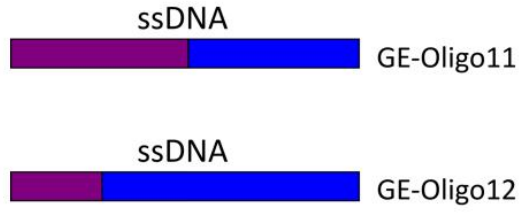

C sgRnA 1

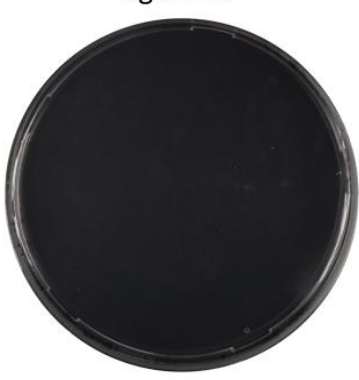

D

E
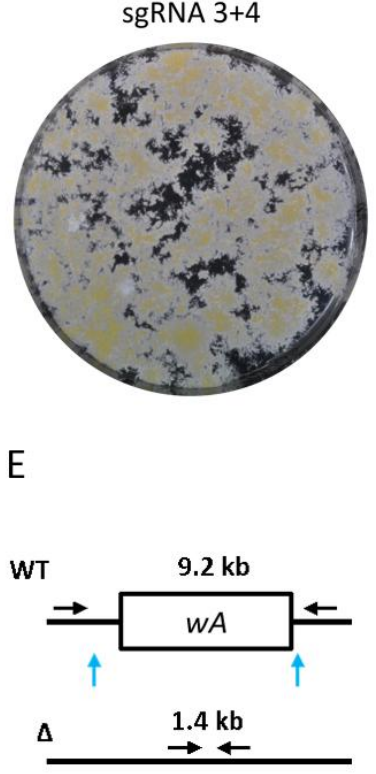

sgRNA 3

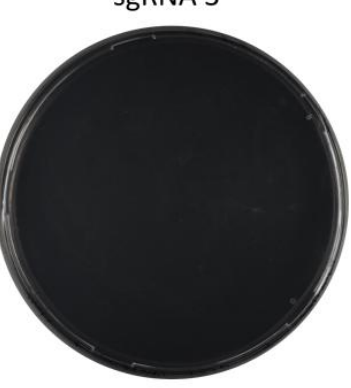

F

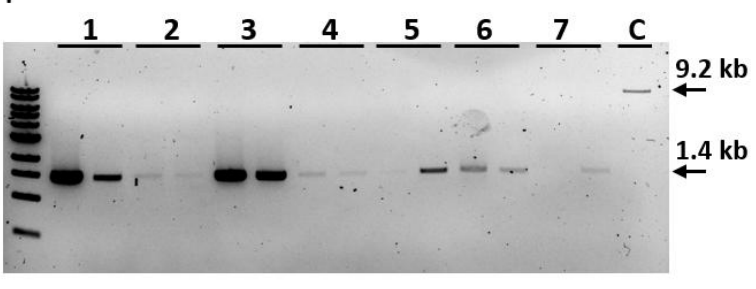

SgRNA 4

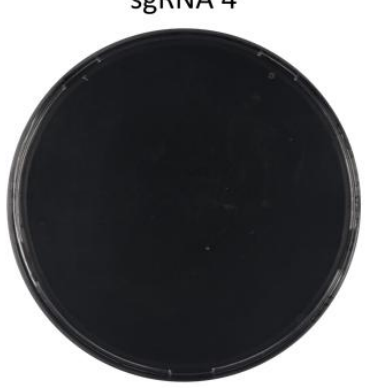

$9.2 \mathrm{~kb}$

.4 kb

A

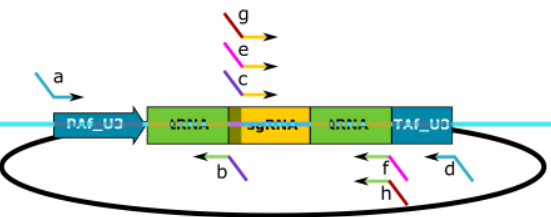

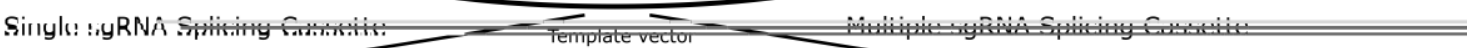
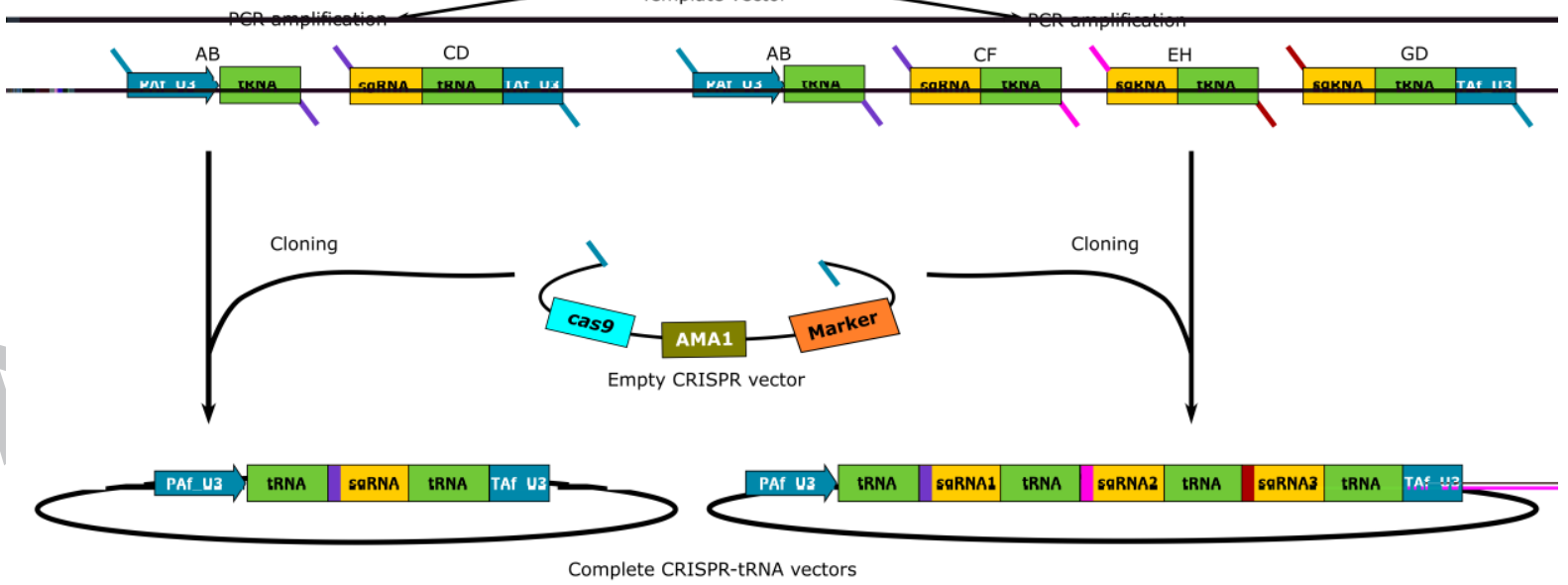

B

GGGTTTAAU PAf_U3

UUnnnnnnA

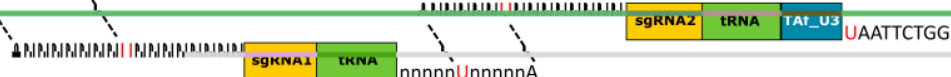



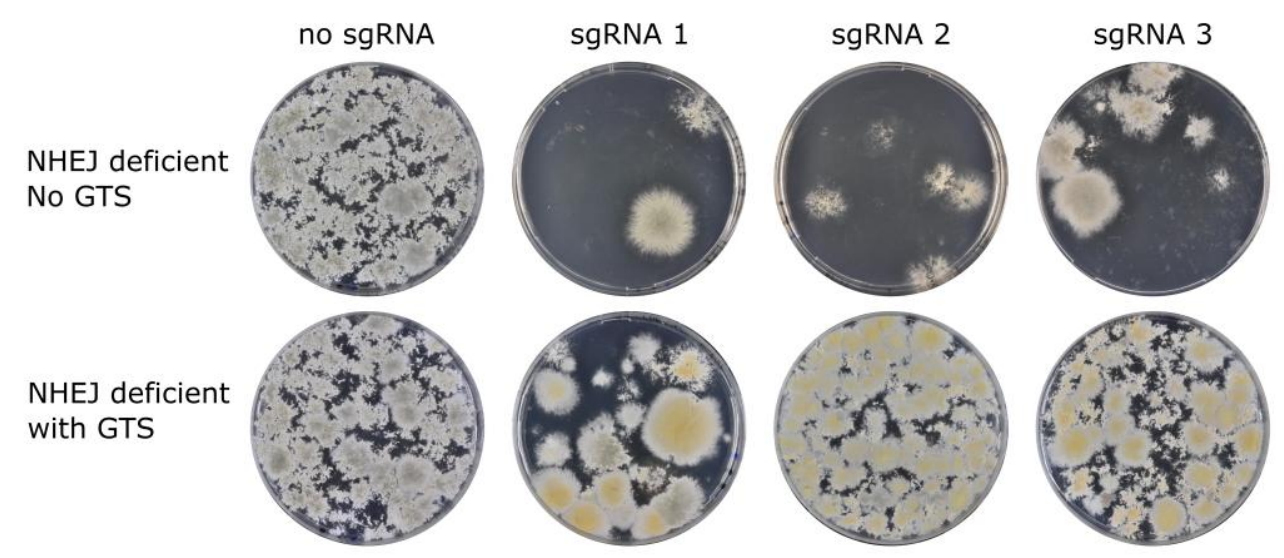

sgRNA 4

NHEJ deficient
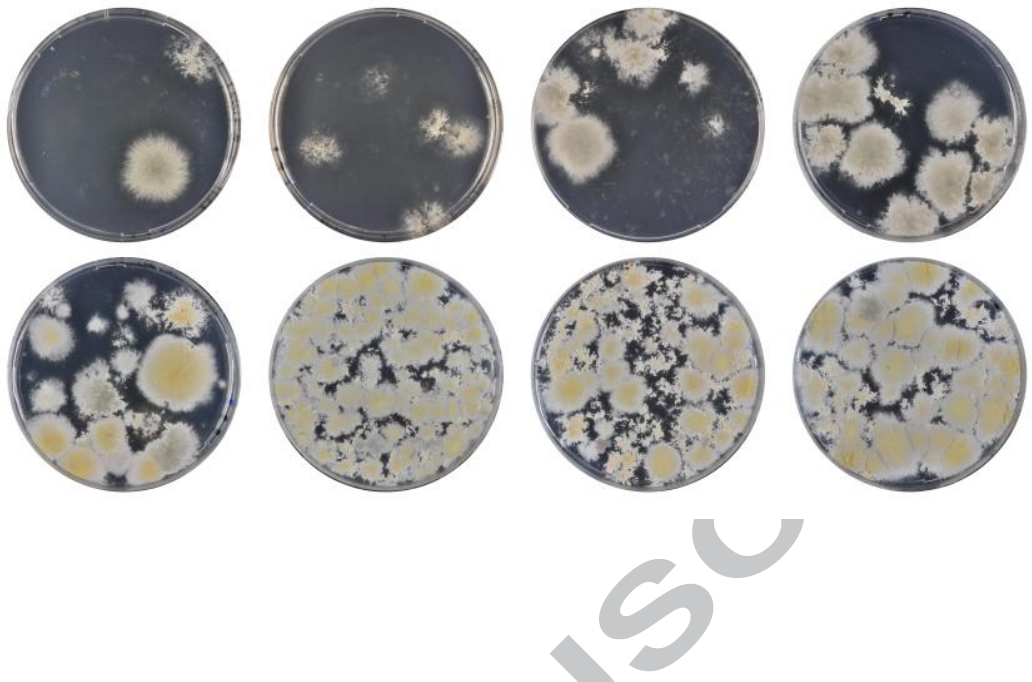
Table 1. Strains used in this study.

\begin{tabular}{|c|c|c|c|}
\hline Strain & Species & Genotype & Source \\
\hline NID1 & Aspergillus nidulans & \multirow{2}{*}{$\begin{array}{l}\text { veA1, } \arg B 2, \text { pyrG89, } \\
n k u A \Delta\end{array}$} & \multirow{2}{*}{ Nielsen et al., 2008} \\
\hline & & & \\
\hline NID5 & Aspergillus nidulans & veA1, $\operatorname{argB2}$, pyrG89 & IBT 27263 \\
\hline NIG1 & Aspergillus niger & - & ATCC1015 \\
\hline NIG81 & Aspergillus niger & pyrG1 & $\begin{array}{l}\text { This study } \\
\text { (Supplementary) }\end{array}$ \\
\hline NIG96 & Aspergillus niger & pyrG1, kusA & $\begin{array}{l}\text { This study } \\
\text { (Supplementary) }\end{array}$ \\
\hline PFJo218 & Aspergillus oryzae & pyrG $\Delta, k u 70 \Delta$ & \multirow{2}{*}{$\begin{array}{l}\text { patent } \\
\text { W008138835 }\end{array}$} \\
\hline & & & \\
\hline
\end{tabular}

${ }^{a}$ A modification of $A$. oryzae NBRC4177, which is available from Institute for fermentation, Osaka; 17-25 Juso Hammachi 2-Chome Yodogawa-Ku. 
Table 2. Gene-targeting efficiency depends on homology length of the gene-targeting substrate.

\begin{tabular}{|c|c|c|c|c|c|}
\hline Experiment & $\begin{array}{c}\text { cas9- } \\
\text { Plasmid } \\
\text { Marker }\end{array}$ & $\begin{array}{c}\text { GTS } \\
\text { Design }\end{array}$ & $\begin{array}{l}\text { Length } 0 \\
\text { Targeting } \\
\text { Sequence } \\
\text { (bps) }{ }^{\mathrm{a}}\end{array}$ & $\begin{array}{c}\text { Number of } \\
\text { Transformants } \\
\text { Yellow (Total) }\end{array}$ & $\begin{array}{c}\text { Average } \\
\text { Gene- } \\
\text { Targeting } \\
\text { Efficiency } \\
\text { (\%) }\end{array}$ \\
\hline 1 & & & 200 & $2(19) ; 1(5)$ & 13 \\
\hline 2 & & & 500 & $7(8) ; 9(18) ; 2(2)$ & 64 \\
\hline 3 & & & 1000 & $54(58)$ & 93 \\
\hline 4 & & & 2000 & $69(75)$ & 92 \\
\hline 5 & & & 200 & $1(5) ; 1(16)$ & 10 \\
\hline 6 & & & 500 & $2(11) ; 4(27)$ & 16 \\
\hline 7 & & & 1000 & $5(16) ; 17(44)$ & 37 \\
\hline 8 & & & 2000 & $14(24)$ & 59 \\
\hline 9 & & & 200 & $5(5) ; 4(4) ; 0(0) ; 9(15)$ & 75 \\
\hline 10 & & $\boldsymbol{A}$ & 500 & $10(10): 12(12)$ & 100 \\
\hline 11 & & & 1000 & $13(13) ; 18(18)$ & 100 \\
\hline 12 & & & 2000 & $8(8) ; 24(26)$ & 94 \\
\hline 13 & pyrG & Linear & 200 & $\begin{array}{c}0(0) ; 0(0) ; 0(0) ; 0(0) \\
0(5) ; 1(1)\end{array}$ & 17 \\
\hline 14 & & & 500 & $\begin{array}{c}2(2) ; 3(3) ; 3(3) ; \\
38(41)\end{array}$ & 94 \\
\hline 15 & & & 1000 & $6(6) ; 11(11) ; 8(10)$ & 93 \\
\hline 16 & & & 2000 & $25(27)$ & 93 \\
\hline
\end{tabular}


${ }^{a}$ The number represents the length of the up-stream as well as of the down-stream targeting sequences of a given gene-targeting substrate. 


\section{Highlights}

- A set of tools for efficient CRISPR-Cas9 based gene editing is developed and successfully used in three different species of Aspergillus

- A tool, TAPE, which allows identification of efficient protospacers is implemented

- tRNAs are used as ribozyme substrates to release sgRNA species from a single transcript setting the stage for multiplex experiments

- Demonstrating that single-stranded oligonucleotides can be used to introduce markerfree deletions and point mutations into the genome with very high efficiencies.

- Demonstrating that oligonucleotides and gene-targeting substrates can be successfully used in combination in multiplex experiment $\mathrm{v}$ 